\title{
LEAFY COTYLEDON2-mediated control of the endogenous hormone content: implications for the induction of somatic embryogenesis in Arabidopsis
}

\author{
Barbara Wójcikowska • Małgorzata D. Gaj
}

Received: 10 August 2014/ Accepted: 9 December 2014/Published online: 17 December 2014

(C) The Author(s) 2014. This article is published with open access at Springerlink.com

\begin{abstract}
The overexpression of LEAFY COTYLEDON2 (LEC2) in Arabidopsis, results in the induction of somatic embryogenesis (SE) in an auxin-free environment and the stimulation of auxin biosynthesis was postulated as being involved in this response. To gain further insight into the hormone-related functions of $L E C 2$ in $\mathrm{SE}$, the effect of $L E C 2$ overexpression on the hormone content in Arabidopsis plants and in vitro cultured explants was analysed. In addition to indole-3-acetic acid (IAA) and cytokinins (CKs), which are hormones that play a key role in plant development, the stress-related hormones, abscisic acid (ABA) and salicylic acid (SA), which are involved in the stress response that is related to SE-induction, were analysed. Together with the observations that LEC2 activity can compensate for the auxin treatment required for $\mathrm{SE}$ induction (Ledwoń and Gaj in Plant Tissue Cell Org Cult 28:1677-1688, 2009) and LEC2 may control auxin biosynthesis pathway during SE induction (Wójcikowska et al. in Planta 238:425-440, 2013), a significant increase in the IAA content in response to $L E C 2$ overexpression found in the present study supply further evidence that LEC2-controlled auxin biosynthesis may be involved in the mechanism that triggers embryogenic development in somatic cells. Moreover, LEC2-controlled SE induction was shown to be associated with a decrease in the total content of CKs and an accumulation of some specific CK types, including isopentenyl-adenin and cis-zeatin. Additionally, an increase in SA and a decrease in ABA content were also found to be related to $L E C 2$ activity in embryogenically induced tissue. The obtained results provide further proof
\end{abstract}

B. Wójcikowska $(\bowtie) \cdot$ M. D. Gaj

Department of Genetics, University of Silesia, ul. Jagiellońska

28, 40-032 Katowice, Poland

e-mail: bwojcikowska@us.edu.pl of the close link between LEC2 and the establishment of the hormonal environment that is required for the promotion of SE.

Keywords Auxin $\cdot$ Cytokinins $\cdot \mathrm{ABA} \cdot \mathrm{SA} \cdot$ LEC2 overexpression $\cdot$ In vitro culture

$\begin{array}{ll}\text { Abbreviations } \\ \text { 2,4-D } & \text { 2,4-Dichlorophenoxyacetic acid } \\ \text { ABA } & \text { Abscisic acid } \\ \text { CKs } & \text { Cytokinins } \\ \text { cZ } & \text { cis-zeatin } \\ \text { DEX } & \text { Dexamethasone } \\ \text { DZR } & \text { Dihydrozeatin-ribosid } \\ \text { FW } & \text { Fresh weight } \\ \text { IAA } & \text { Indolilic-3-acetic acid } \\ \text { iP } & \text { Isopentenyl-adenin } \\ \text { IZE } & \text { Immature zygotic embryo } \\ \text { LEC2 } & \text { LEAFY COTYLEDON2 } \\ \text { SA } & \text { Salicylic acid } \\ \text { SE } & \text { Somatic embryogenesis } \\ \text { tZ7G } & \text { trans-zeatin-7-glucoside } \\ \text { tZ9G } & \text { trans-zeatin-9-glucoside } \\ \text { tZROG } & \text { trans-zeatin-riboside- } O \text {-glucosid } \\ \text { ZR } & \text { trans-zeatin-ribosid }\end{array}$

The LEC2 gene, which is a master regulator of zygotic embryogenesis in Arabidopsis, has been suggested as being in control of embryogenic development in somatic cells and the SE-promoting effect of $L E C 2$ overexpression in plants and explants cultured under auxin-free conditions was reported (Stone et al. 2001; Ledwoń and Gaj 2009). Hence, the overexpression of LEC2 was postulated as 
compensating for the auxin treatment and accordingly, $L E C 2$-stimulated expression of the YUC (YUCCA) genes, which encode the key enzymes in tryptophan dependent pathway of auxin biosynthesis, was recently indicated in SE (Wójcikowska et al. 2013).

To gain further insight into the auxin-related function of $L E C 2$ in SE, we evaluated the effect of $L E C 2$ overexpression on the IAA content in: (1) plants that are able to produce somatic embryos in planta and (2) immature zygotic embryo (IZE) explants that were cultured in vitro on auxin medium. Considering the fact that the extensive interactions between auxin and other hormones are associated with plant development and that a variety of hormone-related genes were reported to be LEC2-controlled (Braybrook et al. 2006), we found it to be reasonable to also analyse the CKs, which also play an essential role in plant development and the stress hormones (ABA and SA), which are believed to affect in vitro induced SE.

Transgenic plants of Arabidopsis thaliana (L.) Heynh., which carry a 35S::LEC2-GR transgene, were used in the study (Ledwoń and Gaj 2009). The plants display a high and stable level of the $L E C 2$ transcripts after treatment with $30 \mu \mathrm{M}$ of water soluble dexamethasone (DEX) (Sablowski and Meyerowitz 1998).

The plants that were used as the source of the IZE explants were grown in Jiffy- 7 pots at $22{ }^{\circ} \mathrm{C}$ under a $16 \mathrm{~h}$ photoperiod of $100 \mu \mathrm{M} \mathrm{m}^{-2} \mathrm{~s}^{-1}$ white, fluorescent light. The IZEs were used as explants for the in vitro culture. In order to induce SE, the standard protocol for Arabidopsis was used (Gaj 2001). Accordingly, ten IZEs were cultured in a Petri dish on an agar-solidified $\left(8 \mathrm{~g} \mathrm{~L}^{-1}\right)$ induction medium (E5) containing basal B5 macro and micro-elements (Gamborg et al. 1968), $20 \mathrm{~g} \mathrm{~L}^{-1}$ sucrose and $5 \mu \mathrm{M}$ 2,4-dichlorophenoxyacetic acid (2,4-D) (Sigma). The IZE explants that were cultured for 5 days on an E5 medium were used for HLPC analysis.

To produce plants for induction of SE in planta, seeds of the 35S::LEC2-GR transgenic line were sterilised in a $20 \%$ solution of commercial bleach for $20 \mathrm{~min}$ and then rinsed three times in sterile water. Seeds were then germinated on a 1/2 MS medium (Murashige and Skoog 1962) containing $10 \mathrm{~g} \mathrm{~L}^{-1}$ sucrose and $8 \mathrm{~g} \mathrm{~L}^{-1}$ agar. Four-weekold plants that produced somatic embryos after DEXtreatment were used for the HLPC analysis.

Plants and IZE-cultures were grown at $23{ }^{\circ} \mathrm{C}$ under a $16 \mathrm{~h}$ photoperiod of $40 \mu \mathrm{M} \mathrm{m}^{-2} \mathrm{~s}^{-1}$ white, fluorescent light.

HPLC analysis was used to evaluate the content of different hormones including: indole-3-acetic acid (IAA), abscisic acid (ABA), salicylic acid (SA) as well as ten forms of cytokinins: trans-zeatin-7-glucoside (tZ7G), trans-zeatin-9-glucoside (tZ9G), cis-zeatin (cZ), transzeatin-riboside- $O$-glucosid (tZROG), dihydrozeatin-ribosid (DZR), trans-zeatin-ribosid (ZR) and isopentenyl-adenin
(iP), trans-zeatin (tZ), dihydrozeatin (DHZ) and transzeatin- $O$-glucosid (tZOG). Hormones were extracted from tissue that had been frozen in liquid nitrogen and $100 \mathrm{mg}$ of tissue was used per sample. The plant tissues for HPLC analysis were produced in two biological replicates, and two technical replicates of each repetition were carried out. HPLC analysis followed the method described by Großkinsky et al. (2014) and was performed in the Department of Plant Physiology, Institute of Plant Sciences, University of Graz, Austria.

Statistical analyses were performed using the student's $t$ test embedded in microsoft excel. Only a return of $P<0.05$ was designated as being statistically significant.

The HLPC analysis indicated a significant modulation in the hormone content and profile in tissue that overexpressed $L E C 2$. A dramatic increase in the IAA content in the plants and IZE-culture in response to $L E C 2$ overexpression was observed. The level of IAA, which was undetected in the control plants, increased significantly in the DEXtreated plants that produced somatic embryos (Fig. 1a). A significant, more than a three-fold increase in the IAA content was also observed in the transgenic IZE-culture that were induced on the auxin medium and treated with DEX. This result supports our earlier postulation that the reduced SE efficiency and prominent callus formation that was observed in the transgenic IZEs cultured on auxinmedium results from over-optimal for SE induction production of IAA (Ledwoń and Gaj 2009). A substantially higher IAA level was accumulated in the plants $\left(508.7 \mathrm{ng} \mathrm{g}^{-1} \mathrm{FW}\right)$ than in the IZE-culture $\left(29.8 \mathrm{ng} \mathrm{g}^{-1}\right.$ FW), which possibly results from the tissue-specific genetic components and regulatory pathways that are involved in the LEC2-controlled auxin biosynthesis (Zhao 2010). In addition, exogenous 2,4-D, which was used in the IZE culture to induce SE, may strongly affect the endogenous IAA accumulation (Pasternak et al. 2002). Thus, there may be some differences in the auxin-related mechanisms that are involved in SE induction in planta (plants) and in vitro (auxin-cultured IZEs).

Out of the ten CK-types that were analysed, three (tZ, $\mathrm{DH}$ and $\mathrm{ZOG}$ ) were not detected in any of the tissue samples. The analysis showed that in contrast to auxin, $L E C 2$ overexpression negatively affected the level of CKs in both the plants and the IZE-culture (Fig. 1b). An LEC2induced reduction in the total CK content was especially dramatic in plants (2.5-fold) and was coupled with a considerably high IAA accumulation. LEC2 overexpression was also found to significantly limit the diversity of the CK-types that accumulated. Three of the CKs (tZ9G, tZROG, ZR) were not detected in the DEX-treated tissue while the level of two others (tZ7G, DZR) was found to be significantly reduced in response to $L E C 2$ overexpression. In contrast, the content of $\mathrm{iP}$ and $\mathrm{cZ}$ was found to be 

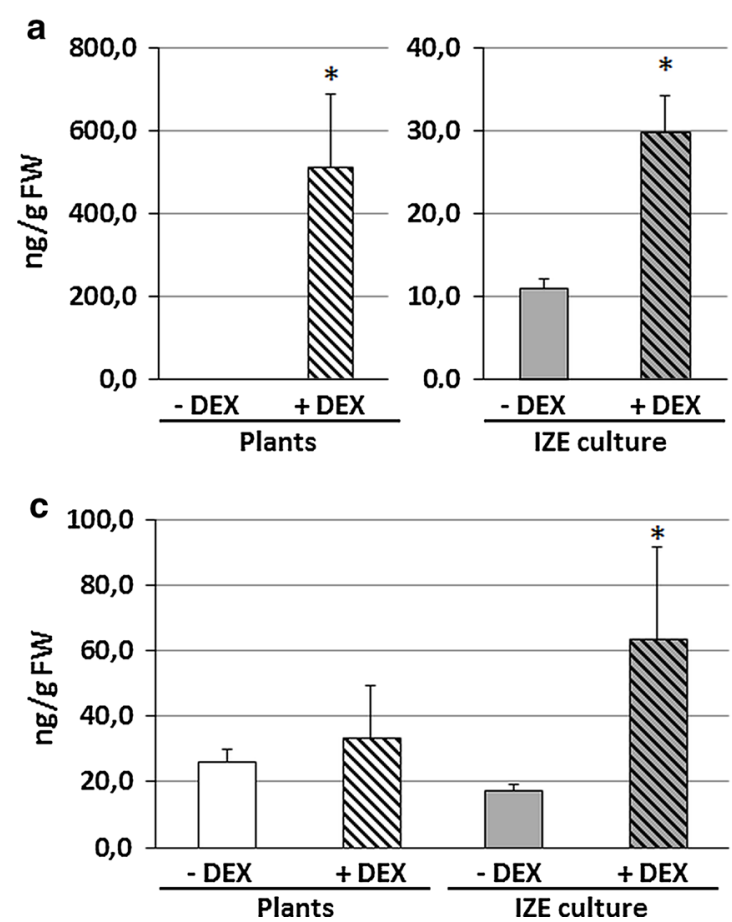

Fig. 1 Level of hormones in plants and IZE-culture of 35S::LEC2GR transgenic line in response to DEX-induced (+DEX) overexpression of $L E C 2$, including: IAA (a); cytokinins: trans-zeatin-7glucoside (tZ7G), trans-zeatin-9-glucoside (tZ9G), cis-zeatin (cZ), trans-zeatin-riboside- $O$-glucosid (tZROG), dihydrozeatin-ribosid

increased in both the plants and in the IZE-culture that overexpressed $L E C 2$. These CKs belong to the isoprenoid CKs, which is the most abundant class of CKs and iP is expected to have a higher biological activity than $\mathrm{cZ}$ (Sakakibara 2006). Collectively, the analyses of CKs indicated that although LEC2-directed SE induction is associated with a decrease in total content of CKs specific types of CKs such as iP and cZ may be positively related to the embryogenic response and their biological function in the mechanism of SE-induction remains to be revealed.

The extensive cross-talk between CKs and auxin in plant development (Moubayidin et al. 2009) might account for the significant inhibition of the $\mathrm{CK}$ accumulation that was observed in tissue in which the overexpression of $L E C 2$ enhanced auxin. A modulation in the level of CKs in response to the auxin treatment was demonstrated in different plants, including Arabidopsis (Nordström et al. 2004). In conclusion, a negative relation between CKs content and embryogenic potential of explant tissue seems to exist in Arabidopsis and an LEC2-mediated reduction in the content of CKs may have a beneficial effect on the induction of SE. Similarly, a low level of CKs was reported to be related to a high embryogenic response in some other plants (Rajasekaran et al. 1987; Wenck et al. 1988).

The present analysis indicated that $L E C 2$ overexpression stimulates a high, threefold increase in the SA
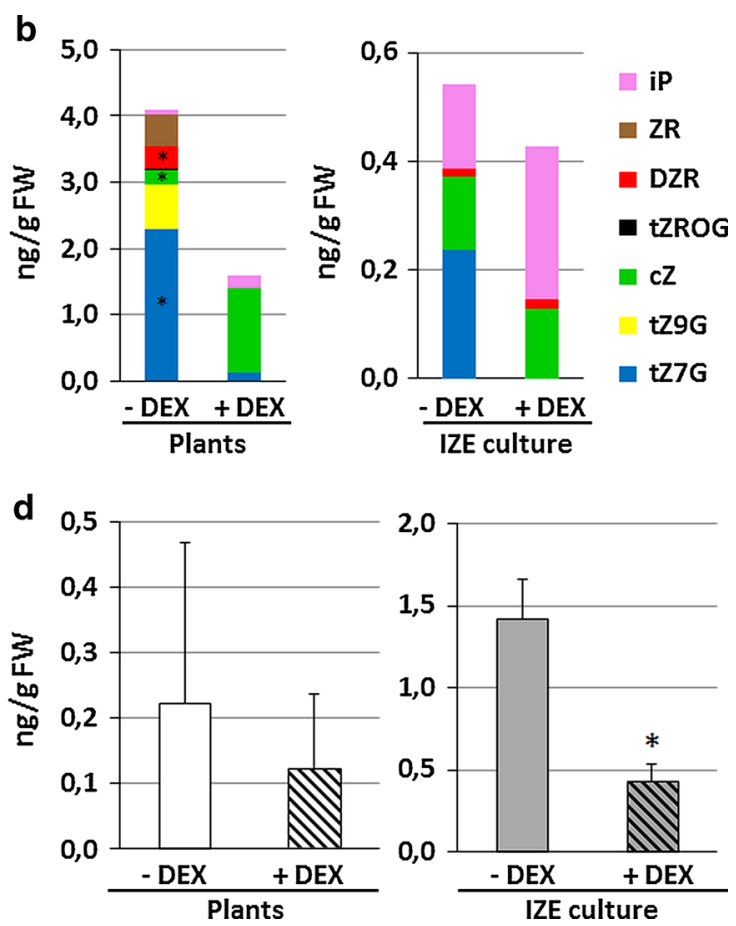

(DZR), trans-zeatin-ribosid (ZR) and isopentenyl-adenin (iP) (b); SA (c) and ABA (d). Values represent the mean \pm SD from two independent sets of samples. Asterisks indicate significant differences between untreated and DEX-treated cultures $(P<0.05$, student's $t$ test). FW, fresh weight

concentration in the IZE culture (Fig. 1c). In contrast, the increase in SA content was not significant in DEX-treated plants implying that SA accumulation in response to $L E C 2$ overexpression seems to be specific to in vitro conditions. This observation suggests that similarly to other plants it can also be assumed that there is a positive relation between SA and embryogenic response in Arabidopsis (Quiroz-Figueroa et al. 2001; Hao et al. 2006). Among the SA-induced mechanisms that promote SE, the inhibition of ethylene production (Roustan et al. 1990) and an increase in the hydrogen peroxide level (Luo et al. 2001), which have positive effect on SE (Kairong et al. 2002), may be considered. The LEC2-stimulation of SA content that was observed may result from an accumulation of AtMES 9 and AtMES12 transcripts of methyl salicylate esterases (MeSA), involved in a SA biosynthesis pathway, that was observed in response to $L E C 2$ overexpression (Braybrook et al. 2006).

The accumulation of ABA in the highly embryogenic cotyledonary stage of the IZEs implied a positive relation between the ABA level and the embryogenic potential of Arabidopsis tissue (Braybrook and Harada 2008). The HPLC results showed a significant reduction in the ABA content in response to $L E C 2$ overexpression and a sharp (45-70 \%) decrease in the ABA level was observed in both the DEX-treated plants and the IZE-culture (Fig. 1d). 
Similarly, a low level of ABA during the initial phases of SE induction was described in other plants (Pescador et al. 2012). In support of the involvement of LEC2 in the reduction of the ABA level during the induction of SE, the genes encoding ABA 8'-hydroxylases (CYP707A1, CYP707A2, CYP707A3) responsible for ABA catabolism were reported to be up-regulated in response to $L E C 2$ overexpression (Braybrook et al. 2006). The presence of the RY motif, which is characteristic of LEC2-controlled genes in a promoter region of the CYP genes further supports the assumption of the LEC2-mediated negative control of ABA activity during SE induction.

One possible scenario of SE induction in Arabidopsis is that the stimulation of $L E C 2$ activity, which can result from the overexpression of the gene (Stone et al. 2001) or 2,4-D treatment (Ledwoń and Gaj 2009), exerts an embryogenic response via the up-regulation of the auxin biosynthesis $Y U C$ genes (Wójcikowska et al. 2013). In response to IAA accumulation, substantial changes in the content and profiles of other hormones, including CKs, SA and ABA (present results), are induced and finally, the hormonecontrolled SE-effectors are triggered. Further experiments are needed to identify the SE-specific targets of the hormone signalling pathways that are involved in the embryogenic transition of somatic cells.

Acknowledgments We thank Dr. Eric van der Graaff (Department of Plant Physiology, Institute of Plant Sciences, University of Graz, Austria) for his help in the HPLC analysis of the hormones and the statistical evaluation of data. The experiments were supported by the Grant of the Rector of the University of Silesia. Author BW was supported by the scholarships within the framework of: the UPGOW project of the European Social Fund, implemented at the University of Silesia and the National Center for Science (Grant No. DEC-2013/ 08/T/NZ2/00800).

Open Access This article is distributed under the terms of the Creative Commons Attribution License which permits any use, distribution, and reproduction in any medium, provided the original author(s) and the source are credited.

\section{References}

Braybrook SA, Harada JJ (2008) LECs go crazy in embryo development. Trends Plant Sci 13:624-630

Braybrook SA, Stone SL, Park S, Bui AQ, Le BH, Fischer RL, Goldberg $\mathrm{RB}$, Harada JJ (2006) Genes directly regulated by $L E A F Y$ COTYLEDON2 provide insight into the control of embryo maturation and somatic embryogenesis. PNAS 103:3468-3473

Gaj MD (2001) Direct somatic embryogenesis as a rapid and efficient system for in vitro regeneration of Arabidopsis thaliana. Plant Cell Tissue Org Cult 64:39-46

Gamborg OL, Miller RA, Ojima K (1968) Nutrient requirement of suspension culture of soybean root cells. Exp Cell Res 50:151-158

Großkinsky DK, Albacete A, Jammer A, Krbez P, van der Graaff E, Pfeifhofer H, Roitsch T (2014) Rapid phytohormone and phytoalexin screening method for physiological phenotyping. Mol Plant 7:1053-1056

Hao L, Zhou L, Xu X, Cao J, Xi T (2006) The role of salicylic acid and carrot embryogenic callus extracts in somatic embryogenesis of naked oat (Avena nuda). Plant Cell Tissue Org Cult 85:109-113

Kairong C, Ji L, Gengmei X, Jianlong L, Lihong W, Yafu W (2002) Effect of hydrogen peroxide on synthesis of proteins during somatic embryogenesis in Lycium barbarum. Plant Cell Tiss Organ Cult 68:187-193

Ledwoń A, Gaj MD (2009) LEAFY COTYLEDON2 gene expression and auxin treatment in relation to embryogenic capacity of Arabidopsis somatic cells. Plant Cell Tissue Org Cult 28:1677-1688

Luo JP, Jiang ST, Pan LJ (2001) Enhanced somatic embryogenesis by salicylic acid of Astragalus adsurgens Pall.: relationship with $\mathrm{H}_{2} \mathrm{O}_{2}$ production and $\mathrm{H}_{2} \mathrm{O}_{2}$-metabolizing enzyme activities. Plant Sci 161:125-132

Moubayidin L, Di Mambro R, Sabatini S (2009) Cytokinin-auxin crosstalk. Trends Plant Sci 14:557-562

Murashige T, Skoog FA (1962) A revised medium for rapid growth and bioassays with tobacco tissue cultures. Physiol Plant 15:473-497

Nordström A, Tarkowski P, Tarkowska D, Norbaek R, Astot C, Dolezal K, Sandberg G (2004) Auxin regulation of cytokinin biosynthesis in Arabidopsis thaliana: a factor of potential importance for auxin-cytokinin-regulated development. PNAS 101:8039-8044

Pasternak T, Prinsen E, Ayaydin F, Miskolczi P, Potters G, Asard H, van Onckelen H, Dudits D, Feher A (2002) The role of auxin, pH and stress in the activation of embryogenic cell division in leaf protoplast-derived cells of alfalfa. Plant Physiol 129:1807-1819

Pescador R, Kerbauy GB, de Melo Ferreira, Purgatto E, Suzuki RM, Guerra MP (2012) A hormonal misunderstanding in Acca sellowiana embryogenesis: levels of zygotic embryogenesis do not match those of somatic embryogenesis. Plant Growth Regul 68:67-76

Quiroz-Figueroa F, Mendez-Zeel M, Larque-Saavedra A, LoyolaVargas VM (2001) Picomolar concentrations of salicylates induce cellular growth and enhance somatic embryogenesis in Coffea arabica tissue culture. Plant Cell Rep 20:679-684

Rajasekaran K, Hein MB, Vasil IK (1987) Endogenous abscisic acid and indole-3-acetic acid and somatic embryogenesis in cultured leaf explants of Pennisetum purpureum Schum. Plant Physiol $84: 47-51$

Roustan JP, Latche A, Fallot J (1990) Inhibition of ethylene production and stimulation of carrot somatic embryogenesis by salicylic acid. Biol Plant 32:273-276

Sablowski RWM, Meyerowitz EM (1998) A homolog of NO APICAL $M E R I S T E M$ is an immediate target of the floral homeotic genes APETALA3/PISTILLATA. Cell 92:93-110

Sakakibara H (2006) Cytokinins: activity, biosynthesis and translocation. Annu Rev Plant Biol 57:431-449

Stone SL, Kwong LW, Yee KM, Pelletier J, Lepiniec L, Fischer RL, Goldberg RB, Harada JJ (2001) LEAFY COTYLEDON2 encodes a B3 domain transcription factor that induces embryo development. PNAS 98:11806-11811

Wenck AR, Conger BV, Trigiano RN, Sams CE (1988) Inhibition of somatic embryogenesis in orchard grass by endogenous cytokinins. Plant Physiol 88:990-992

Wójcikowska B, Jaskóła K, Gąsiorek P, Meus M, Nowak K, Gaj MD (2013) LEAFY COTYLEDON2 (LEC2) promotes embryogenic induction in somatic tissues of Arabidopsis, via YUCCAmediated auxin biosynthesis. Planta 238:425-440

Zhao Y (2010) Auxin biosynthesis and its role in plant development. Annu Rev Plant Biol 61:49-64 